\title{
SEP Montage Variability Comparison during Intraoperative Neurophysiologic Monitoring
}

\author{
Christine Hanson, Athena Maria Lolis and Aleksandar Beric*
}

Department of Neurology, Division of Clinical Neurophysiology, New York University School of Medicine, New York, NY, USA

\section{OPEN ACCESS}

Edited by:

A. Arturo Leis,

Mayo Clinic Arizona, USA;

Methodist Rehabilitation Center, USA

Reviewed by:

D. Stokic,

Methodist Rehabilitation Center, USA

William Joseph Triggs,

University of Florida, USA

*Correspondence:

Aleksandar Beric

aberic1@hotmail.com

Specialty section:

This article was submitted to

Neuromuscular Diseases,

a section of the journal

Frontiers in Neurology

Received: 04 January 2016 Accepted: 20 June 2016

Published: 30 June 2016

Citation:

Hanson C, Lolis AM and Beric A (2016) SEP Montage Variability

Comparison during Intraoperative

Neurophysiologic Monitoring.

Front. Neurol. 7:105.

doi: 10.3389/fneur.2016.00105
Intraoperative monitoring is performed to provide real-time assessment of the neural structures that can be at risk during spinal surgery. Somatosensory evoked potentials (SEPs) are the most commonly used modality for intraoperative monitoring. SEP stability can be affected by many factors during the surgery. This study is a prospective review of SEP recordings obtained during intraoperative monitoring of instrumented spinal surgeries that were performed for chronic underlying neurologic and neuromuscular conditions, such as scoliosis, myelopathy, and spinal stenosis. We analyzed multiple montages at the baseline, and then followed their development throughout the procedure. Our intention was to examine the stability of the SEP recordings throughout the surgical procedure on multiple montages of cortical SEP recordings, with the goal of identifying the appropriate combination of the least number of montages that gives the highest yield of monitorable surgeries. Our study shows that it is necessary to have multiple montages for SEP recordings, as it reduces the number of non-monitorable cases, improves IOM reliability, and therefore could reduce false positives warnings to the surgeons. Out of all the typical montages available for use, our study has shown that the recording montage $\mathrm{Cz}-\mathrm{C} 4 / \mathrm{Cz}-\mathrm{C} 3(\mathrm{Cz}-\mathrm{Cc})$ is the most reliable and stable throughout the procedure and should be the preferred montage followed throughout the surgery.

Keywords: IOM, SEP, IOM recording montages, montage comparison, spine surgery, SEP reliability

\section{INTRODUCTION}

The ultimate goal of intraoperative neurophysiological monitoring (IOM) is to reduce the risk of adverse events that can occur during surgeries that put neural structures at risk in an attempt to prevent permanent neurological injury. Somatosensory evoked potentials (SEP) have been used for IOM since their inception in the late 60s and 70s (1-5). Through the years, the field of IOM has been improved by the addition of other modalities, such as electromyography (EMG) and motor evoked potentials (MEP); however, SEP recording have continued to remain the gold standard. False-positive SEP changes during surgery can transiently occur due to various causes, such as change in anesthesia delivery, blood loss, blood pressure, body temperature, accumulation of anesthetic in the patient's body, and various technical interferences in the operating room. For the purposes of conducting IOM, it is very important to distinguish real SEP changes from sources of interference, such as technical problems or noise, which may lead to an increase in number of false positive changes. 
Somatosensory evoked potentials are usually recorded with multichannel derivations of cortical and subcortical responses. $\mathrm{Cz}^{\prime}, \mathrm{C}^{\prime}$ ', and $\mathrm{C4}^{\prime}$ are situated according to the modified 10-20 system for EEG electrode placement: $\mathrm{Cz}^{\prime}$ is located $2 \mathrm{~cm}$ posterior to $\mathrm{Cz}, \mathrm{C}^{\prime}$ (left) and $\mathrm{C}^{\prime}$ (right) $7 \mathrm{~cm}$ from $\mathrm{Cz}^{\prime}$ on a line connecting it with the external auditory meatus. For the purposes of this study, $\mathrm{Cz}^{\prime}$ will be referred to as $\mathrm{Cz}$. $\mathrm{C}^{\prime}$ and $\mathrm{C4}^{\prime}$ will be referred to as $\mathrm{Cc}$, if contralateral to stimulated extremity and $\mathrm{Ci}$ when ipsilateral to stimulated extremity in this article. The locations of these traditional recording electrodes $\mathrm{Cc}$ (upper extremity) and $\mathrm{Cz}$ (lower extremity) represent the location of the arm and leg area primary somatosensory cortex within the postcentral gyrus. $\mathrm{Cz}$ can be used bilaterally for recording of tibial and peroneal SEPs since the leg areas are located in depth of the interhemispheric fissure on the postcentral gyrus very close to one another. The $\mathrm{C}^{\prime} \mathrm{Fz} / \mathrm{C} 4^{\prime} \mathrm{Fz}$ is the montage that is typically used for obtaining the cortical responses of the upper extremity. Since the first description of peroneal nerve SEPs and tibial nerve SEPs, $\mathrm{Cz}^{\prime} \mathrm{Fz}$ is the montage used for SEP recording of lower extremity responses $(6,7)$.

Beric and Prevec in 1981 discovered a peculiar tibial nerve cortical generator behavior and in 1983 published that the tibial nerve SEP exhibits asymmetrical scalp amplitude distribution $(8,9)$. The positively charged sensory leg generator is situated slightly lateral to $\mathrm{Cz}$ in the hemisphere contralateral to the stimulated leg. Isopotential lines around it appear to be distorted toward the hemisphere ipsilateral to the stimulated leg. Therefore, $\mathrm{Ci}$ is partially active (positive) while $\mathrm{Cc}$ appears to represent a negatively charged area. The question whether this is due to the presence of at least two generators or to the existence of a dipole (9-16) has yet to be resolved. The responses of tibial and peroneal nerves are historically recorded in analogy to the location of the recording electrodes for median and ulnar SEPs. Fz is used as reference and $\mathrm{Cz}$, representing the leg area on the somatosensory cortex, as recording electrode. Interestingly, the distribution of potentials evoked by stimulation of the tibial and peroneal nerves suggests $\mathrm{CiCc}$ and $\mathrm{CzCc}$ as good possible montage alternatives to $\mathrm{CzFz}$. This can be achieved using the preexisting electrodes already placed for routine upper and lower extremity SEP monitoring. $\mathrm{Cz}$ is positively charged, $\mathrm{Ci}$ partially active (positive), $\mathrm{Cc}$ negatively charged, and $\mathrm{Fz}$ practically inactive. Hence, in theory, the amplitude of $\mathrm{P} 40$ for $\mathrm{CiCc}$ should be in the same range as in the $\mathrm{CzFz}$ derivation. For the $\mathrm{CzCc}$ montage, the difference between the two electrodes becomes even more positive over a differential amplifier; hence, P40 amplitude would here be expected to be the largest.

Most default IOM machine protocols and most surgical centers seem to be using $\mathrm{CzFz}$ or $\mathrm{CiCc}$ montages for cortical IOM traces. Existing guidelines by the American Clinical Neurophysiology Society and by the American Society of Neurophysiological Monitoring suggest that the use of multichannel recording should be performed while conducting IOM in case of technical problems. According to the guidelines, different derivations, such as $\mathrm{CiCc}$ and $\mathrm{CzCc}$, should be considered in order to determine and select the trace with the highest amplitude (17-19). However, the selection of these montages at baseline does not take into account the development of the recordings throughout the surgery. The goal of this study was to find the most appropriate combination of the least number of montages that gives the highest yield of monitorable surgeries. We wanted to analyze and compare these three montages, $\mathrm{CzFz}, \mathrm{CiCc}$, and $\mathrm{CzCc}$ in regard to their appearance at baseline as well as their development throughout the surgery. Amplitude size is important for SEP IOM, but the consistency during the surgery leading to stable and reliable monitoring avoiding major fluctuations in amplitude and latency is even more important. This especially plays a role during the monitoring if the routinely used montage has lower amplitudes at baseline or shows poorly repeatable baseline recordings. Our study takes this into account, since we believe that possible improvement of SEP reliability during the surgery by using different electrode montages can be of benefit for the patient.

\section{MATERIALS AND METHODS}

This prospective study evaluated 524 consecutive patients undergoing surgery at NYU Langone Medical Center from January 1, 2010 through December 31, 2010 who did not exhibit any postoperative neurologic deficits. Patient's ages ranged from 8 to 84 . All patients had undergone an instrumented spinal fusion surgery with neurophysiologic intraoperative monitoring. Surgical procedures included chronic neurologic and neuromuscular conditions, such as congenital or degenerative spine disease, scoliosis, myelopathy, and spinal stenosis, that were indicated to undergo lumbar spinal fusion, cervical spinal fusion, and scoliosis correction surgery. In all cases, the tibial nerve SEP was followed during IOM, and when indicated, peroneal nerve SEP was also performed. The tibial nerve is the largest distal lower extremity nerve with the greatest representation on the cortex and is used for monitoring of the posterior spinal columns of the spinal cord. The tibial nerve was used for lower extremity SEP monitoring for spinal surgery involving cervical and thoracic levels as well as for monitoring of all surgeries with lumbar spinal instrumentation. For surgery involving level L5 and lower, peroneal nerves were monitored additionally. Three cortical montages $\mathrm{CzFz}, \mathrm{CiCc}$, and $\mathrm{CzCc}$ were set up for lower extremity SEP recording. This is the standard recordings montage and protocol that is routinely performed for instrumented spinal fusions at our institution. At first, 150 patients underwent detailed analysis. After those results were reviewed, another 374 patients were added, using a quicker approach, based on the initial analysis of the first 150 patients. A total of 524 patients with 1048 tibial traces and 546 peroneal traces were assessed.

\section{Stimulation and Recordings}

For stimulation of all nerves, silver-chloride disposable stick-on electrodes were utilized. To stimulate the tibial nerve, the stimulating electrodes were placed behind the medial malleolus. The peroneal nerve was stimulated at the anterolateral aspect of the ankle. For stimulation of both nerves, the cathode was always placed proximally. Nicolet Endeavor CR (Natus, Middleton, WI, USA) was used in all cases of neurophysiologic monitoring. The nerves were stimulated using rectangular pulses of $500 \mu \mathrm{s}$ duration and stimulation frequency was $3.1 \mathrm{~Hz}$. The time base 
was 100 ms. Stimulation intensity was adjusted to get the largest amplitude tracings using the smallest necessary intensity, with the maximal intensity of $35 \mathrm{~mA}$.

The recordings were obtained by using sterile stainless steel subdermal EEG electrodes placed in the scalp. Needles were positioned at $\mathrm{Fz}, \mathrm{Cz}^{\prime}, \mathrm{C}^{\prime}, \mathrm{C}^{\prime}$ according to the modified international 10-20 system for EEG electrode placement. $\mathrm{Cz}^{\prime}$, therefore, was placed above the leg cortical representation area $(1.5-2 \mathrm{~cm}$ behind $\mathrm{Cz}) . \mathrm{C}^{\prime}$ and $\mathrm{C}^{\prime}{ }^{\prime}$ were located above the arm areas $(7 \mathrm{~cm}$ from $\mathrm{Cz}^{\prime}$ on a line connecting it with the external auditory meatus) of the postcentral somatosensory cortex. The resistances were kept below $5 \mathrm{k} \Omega$. Traces were recorded with a $20-1000 \mathrm{~Hz}$ filter and amplified 10,000 times. SEP activity was displayed by the Endeavor software on a Windows PC. Electrode montages set up for recording were $\mathrm{CzFz}, \mathrm{CiCc}$ (Ci being the electrode $\mathrm{C}^{\prime}$ or $\mathrm{C}^{\prime}$ ' ipsilateral to the stimulated leg nerve), and $\mathrm{CzCc}$ (Cc was $\mathrm{C}^{\prime}$ or $\mathrm{C}^{\prime}$ ' above contralateral hemisphere). One hundred responses were averaged. Depending on the stage of the surgery, stimulation was applied every 5-20 min.

\section{Detailed Approach of SEP Analysis}

The first groups of patients were analyzed based on a detailed approach. The first 150 patients, which were 300 tibial and 156 peroneal SEPs recordings, were analyzed in regard to 5 categories: clarity, repeatability, size (amplitude) of response, consistency over the time course of the operation, and level of noise during the procedure. They were given a point for each category, adding up to a score between 5. Clarity, repeatability and size of response were measured at baseline. Consistency and noise (absence of noise) were taken into consideration as they developed throughout the IOM, including the closing trace. Trace in this study is operationally defined as individual montage recording. Criteria for the trace scorings are shown in Table 1. Montages were compared indirectly according to the five categories and their quality for IOM was determined according to their scores (indirect approach).

We considered recordings satisfactory if their scores were 3 and above and present only if their scores were 2 and above. If the trace was well formed, repeatable, with an appropriate amplitude, consistency, and no noise, it was scored 5 in all categories as the example of the traces in Figure 1. As scoring is subjective and the 1-5 scale is not linear and can, therefore, be potentially misleading, the electrode montages were also compared directly (one on one), using the same five categories but given a point only to the better trace in the corresponding category, the other trace was given 0 points (direct approach). This detailed approach was performed in order to assess which relevant component of a single montage is important for quality of IOM and differs from other used montages.

\section{Quick Approach for SEP Analysis}

After the first data collection of SEP recordings was assessed, the remaining 374 patients SEP recordings (748 tibial nerves and 390 peroneal nerves) were analyzed using a quicker approach. This approach involved comparing traces from three different montages directly at Baseline and according to their Development during the case. At the baseline, the clarity, repeatability, and size were assessed as one result. Consistency and noise were evaluated as they developed throughout the case as the second result. This was followed by taking the SEP recordings of the first group of 150 patients, which was analyzed in the detailed approach, and re-assessing the recordings based on the quicker approach described above. At this point, the data of all 524 patients were statistically analyzed as divided into the following pairs for direct comparison: $\mathrm{CzFz}$ vs. $\mathrm{CiCc}, \mathrm{CzFz}$ vs. $\mathrm{CzCc}$, and $\mathrm{CiCc}$ vs. $\mathrm{CzCc}$.

\section{Statistical Analysis}

All data were statistically analyzed using Chi-square tests for direct comparisons of pairs and ANOVA One way tests for comparisons of three groups in indirect montage comparison (detailed approach). We set the significance level $\alpha=1 \%$ in order to achieve more reliable results, thus meaning that only results $p<0.01$ were considered statistically significant. Pearson correlation coefficient was calculated using data from Quick approach only by comparison of baseline traces score vs. development score during the monitoring for each of three montages.

\section{RESULTS}

\section{Indirect and Direct Comparisons of SEPs in Five Categories (Detailed Approach)}

In the first paradigm, 300 tibial nerve recordings and 156 peroneal nerve recordings were analyzed. Each nerve recording used the following montages: $\mathrm{CzFz}, \mathrm{CiCc}$, and $\mathrm{CzCc}$. Each montage was given points in the five categories: clarity, repeatability, size of response, consistency during the monitoring, and noise level throughout the monitoring and added up to a score of $1-5$. Both

TABLE 1 | Criteria for waveform classification of SEP traces in five categories based on a 1-5 scale.

\begin{tabular}{|c|c|c|c|c|c|}
\hline & 1 & 2 & 3 & 4 & 5 \\
\hline Clarity & Absent waveform & $\begin{array}{l}\text { Present waveform, but not } \\
\text { clear P40 and/or N50 }\end{array}$ & Present waveform, clear P40 and N50 & Clearly present entire waveform & Perfect W waveform \\
\hline Repeatability & Not repeatable & Poorly repeatable & Repeatable P40 and N50 & Repeatable entire waveform & Identical waveform \\
\hline Amplitude & $<0.2 \mu \mathrm{V}$ & $0.2-0.4 \mu \mathrm{V}$ & $0.4-0.8 \mu \mathrm{V}$ & $0.8-2.0 \mu \mathrm{V}$ & $>2 \mu \mathrm{V}$ \\
\hline Consistency & $\begin{array}{l}\text { Not present after } \\
\text { surgical opening }\end{array}$ & $\begin{array}{l}\text { Present most of the } \\
\text { time - needs repeated } \\
\text { averaging }\end{array}$ & $\begin{array}{l}\text { Always present, but with variable latency } \\
\text { and/or amplitude - less than alarm criteria }\end{array}$ & $\begin{array}{l}\text { Similar waveform throughout, } \\
\text { same latency and similar } \\
\text { amplitude }\end{array}$ & $\begin{array}{l}\text { Almost identical } \\
\text { waveform } \\
\text { throughout IOM }\end{array}$ \\
\hline Noise & $\begin{array}{l}\text { Noise throughout } \\
\text { IOM }\end{array}$ & $\begin{array}{l}\text { Repeated noise interfering } \\
\text { with waveform recognition }\end{array}$ & $\begin{array}{l}\text { Repeated noise not interfering with } \\
\text { waveform recognition }\end{array}$ & $\begin{array}{l}\text { Occasional noise not interfering } \\
\text { with waveform recognition }\end{array}$ & No noise \\
\hline
\end{tabular}




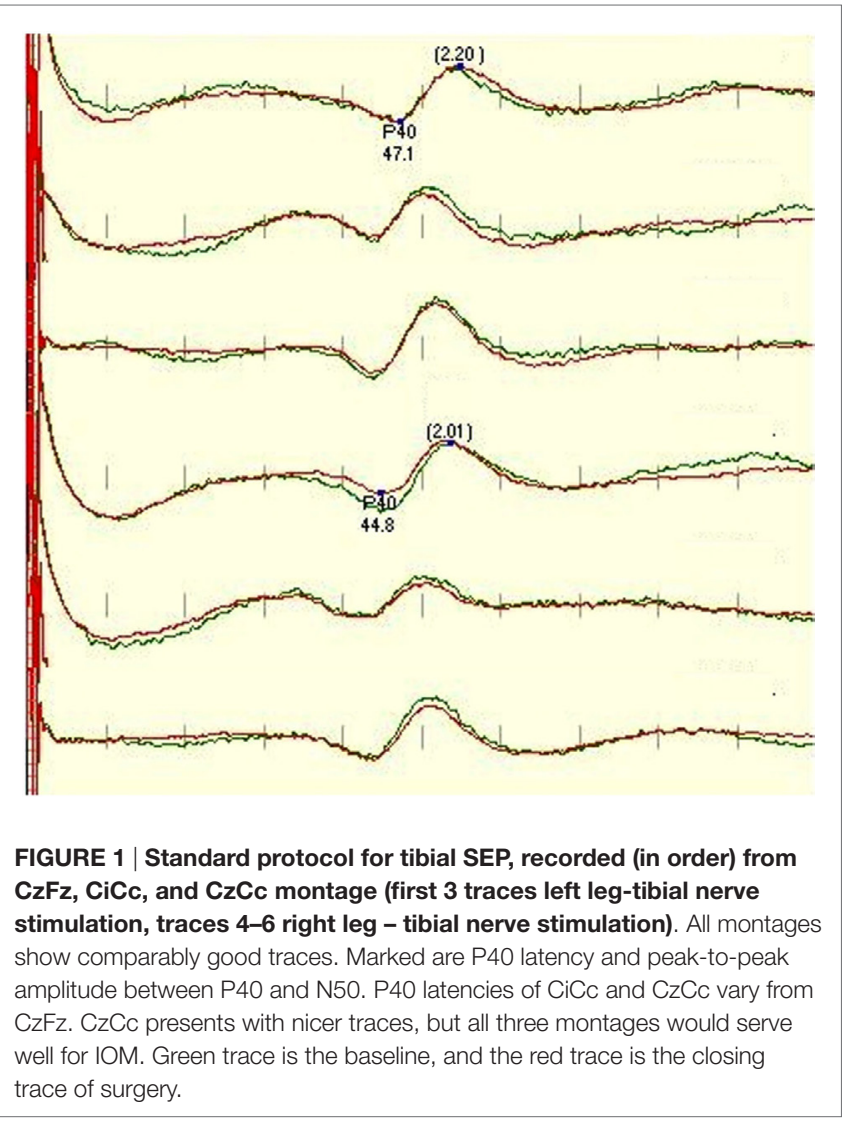

separate indirect ANOVA comparisons were made in all five categories, as well as indirect comparison when all five category scores were averaged.

\section{Indirect Tibial Nerve SEP Montage Comparison}

For tibial monitoring, $\mathrm{CzFz}$ montage showed an average score of 2.82 out of 5 points. Both other montages reached better scores than $\mathrm{CzFz}$, with an average of 3.76 for $\mathrm{CiCc}$ and 3.79 for $\mathrm{CzCc}$ out of 5 points, as shown in Table 2.

There was no statistical difference between $\mathrm{CiCc}$ and $\mathrm{CzCc}$ montage $(p=0.610)$, but both montages were significantly better than $\mathrm{CzFz}$. While there were some response present in 88.7\% of CzFz recordings, $96.3 \%$ of CiCc recordings, and $96.7 \%$ of $\mathrm{CzCc}$ recordings (scores above 2), only $58.0 \%$ of cases had presented with a $\mathrm{CzFz}$ montage that satisfied the intraoperative monitoring (scores $\geq 3$ on $0-5$ scale), as shown in Table 2 . CiCc montage showed $77.3 \%$ and $\mathrm{CzCc}$ recordings showed $79.0 \%$ with scores above 3 , therefore, demonstrating well-monitorable traces in $\sim 20 \%$ more cases than CzFz. For tibial SEPs, CiCc and $\mathrm{CzCc}$ both presented with traces significantly better than $\mathrm{CzFz}$ in all five categories. Figure 2 depicts a high level of noise affecting the $\mathrm{CzFz}$ montage, which prevented this from being monitorable, while the traces from other two montages were practically noise free.

The montages $\mathrm{CiCc}$ and $\mathrm{CzCc}$ showed significant difference only in clarity, as depicted in Figure 3. The differences in the
TABLE 2 | Usability of traces for IOM.

\begin{tabular}{llccc}
\hline & $\begin{array}{c}\text { Median } \\
\text { score out } \\
\text { of } \mathbf{5} \text { points }\end{array}$ & $\begin{array}{c}\text { Traces } \\
\text { non-recordable } \\
\text { or not satisfying } \\
\text { ( } \mathbf{2} \text { on } \mathbf{0 - 5} \text { scale) (\%) }\end{array}$ & $\begin{array}{c}\text { Traces good } \\
\text { for IOM } \\
\text { ( } \mathbf{3} \text { on } \mathbf{0 - 5} \\
\text { scale) (\%) }\end{array}$ \\
\hline Tibial & CzFz & 2.82 & 42.0 & 58.0 \\
$(n=300)$ & CiCc & 3.76 & 22.7 & 77.3 \\
& CzCc & 3.79 & 21.0 & 79 \\
Peroneal & CzFz & 1.64 & 79.2 & 20.8 \\
$(n=146)$ & CiCc & 2.27 & 64.0 & 36.0 \\
& CzCc & 2.66 & 53.0 & 47.0
\end{tabular}

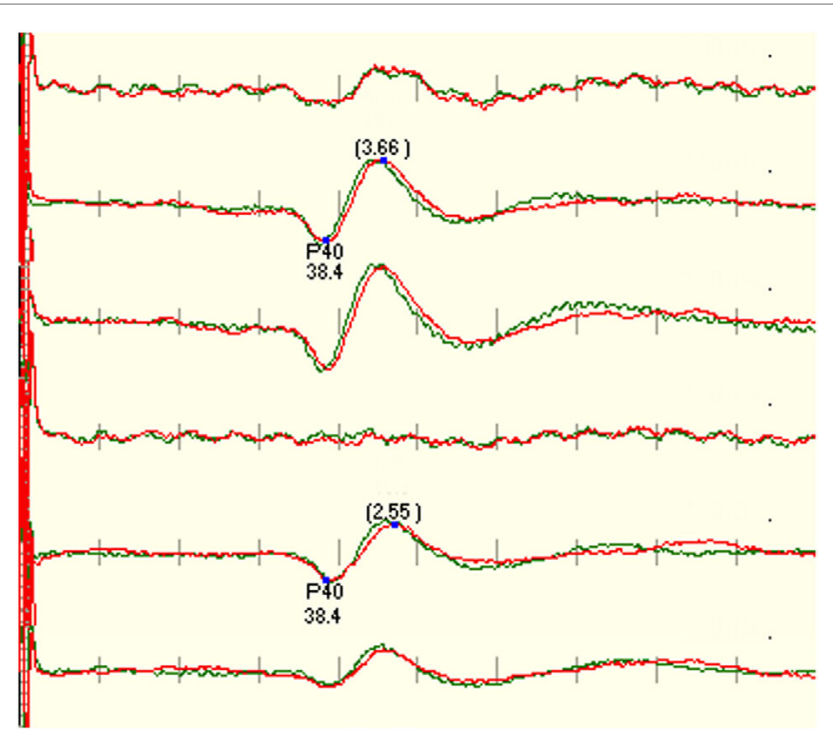

FIGURE 2 | Example that shows the right tibial SEP is not recordable using CzFz montage. Due to good recordings in $\mathrm{CiCc}$ and $\mathrm{CzCc}$, the monitorability of the surgery was improved. CzFz picked up a lot of noise, while the transverse montages are mainly free from noise.

other categories were insignificant. The additional quantitative analysis of size of amplitudes showed no significant difference between the three montages $\mathrm{CzFz}$ (mean $0.91 \mu \mathrm{V}$ ), CiCc $($ mean $0.91 \mu \mathrm{V})$, and $\mathrm{CzCc}($ mean $1.02 \mu \mathrm{V})$. However, $\mathrm{CzCc}$ was the largest.

\section{Direct Tibial Nerve SEP Montages Comparison}

Tibial SEP direct pair comparisons of the montages $\mathrm{CzFz}, \mathrm{CiCc}$, and $\mathrm{CzCc}$ confirm the findings of the indirect observations described above in all five categories as shown in Table 3.

$\mathrm{CiCc}$ montage was better than $\mathrm{CzFz}$ in all categories. These results were significant for repeatability, size, consistency, and noise. CiCc montage showed better results than $\mathrm{CzFz}$; $19.7 \%$ better for repeatability, $26 \%$ for size, and $26.6 \%$ more often better for consistency. It also surpassed $\mathrm{CzFz}$ montage by picking up less noise in 58.6\% more of the IOMs. CzCc proved to be better than $\mathrm{CzFz}$ also in all five categories, and in addition significantly superior for all five categories: $\mathrm{CzCc}$ 


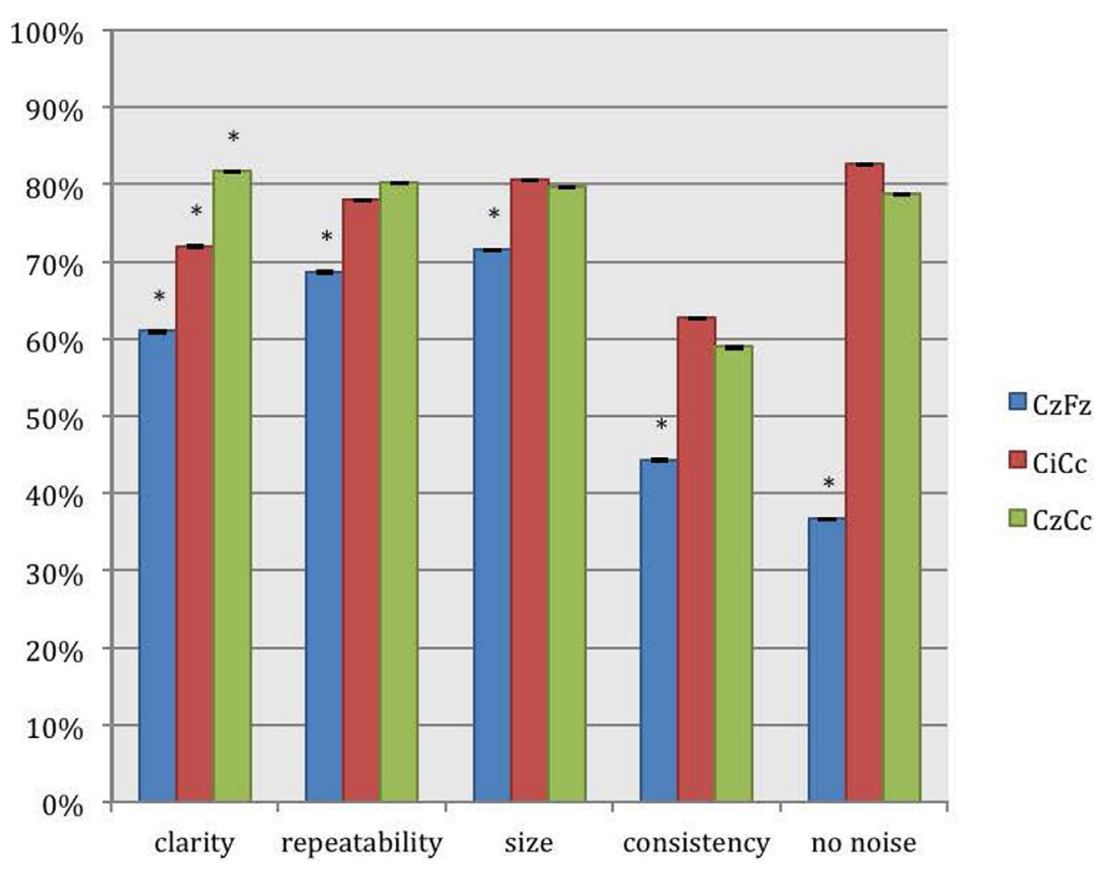

FIGURE 3 | Direct comparison of the montages $\mathrm{CzFz}, \mathrm{CiCc}$, and $\mathrm{CzCc}$ for tibial nerve SEPs according to their score in the five categories: clarity, repeatability, size, consistency, and absence of noise. Asterisks $\left(^{*}\right)$ marks montages significantly different from the other montages. All significance levels (Chi-square tests) were $p<0.001$ with one exception for size of the trace: $\mathrm{CzFz}<\mathrm{CiCc}(p<0.001)$ and $\mathrm{CzFz}<\mathrm{CzCc}(p=0.003)$.

traces presented with clear baselines of $37.6 \%$ more often than $\mathrm{CzFz}$ were better in size $16.7 \%$ more frequent than $\mathrm{CzFz}$, more consistent and repeated better in approximately a third more of the IOMs. Noise was picked up by $\mathrm{CzFz} 49 \%$ more often than by $\mathrm{CzCc}$. As for a comparison between $\mathrm{CiCc}$ and $\mathrm{CzCc}$ in tibial recordings, the two montages showed only little differences. $\mathrm{CzCc}$ was the significantly better montage in $13 \%$ more often than $\mathrm{CiCc}$ for clarity and $6.4 \%$ more often for repeatability. However, CiCc picked up significantly less noise in $5 \%$ more of the IOMs than $\mathrm{CzCc}$.

\section{Indirect Peroneal Nerve SEP Montages Comparison}

The results for peroneal monitoring are similar, and generally all traces had poorer results due to the peroneal nerve being a smaller nerve. $\mathrm{CiCc}$ (2.27) and $\mathrm{CzCc}$ (2.66) montages of the peroneal nerve reached higher scores than the $\mathrm{CzFz}$ (1.64) montage, as shown in Table 2. This result of $\mathrm{CzFz}$ being the weakest montage and $\mathrm{CzCc}$ showing the best traces out of the three montages was statistically significant.

For recordings of the peroneal nerve, $\mathrm{CzCc}$ presented with better traces than $\mathrm{CzFz}$ in all 5 categories, as shown in Figure 4. This result is statistically significant for all categories but size. According to Figure 4, although $\mathrm{CzCc}$ was the significantly better trace according to clarity, repeatability, size $(p=0.036)$, and consistency ( $p=0.004)$, CiCc picked up significantly less noise than $\mathrm{CzCc}(p=0.003)$. While differences between $\mathrm{CzFz}$ and $\mathrm{CiCc}$ were significant only for repeatability and noise, $\mathrm{CiCc}$ also showed a tendency to be the better montage for consistency. The additional quantitative analysis of size of amplitudes showed that there was no significant difference between the three montages $\mathrm{CzFz}$ (mean $0.61 \mu \mathrm{V}), \mathrm{CiCc}($ mean $0.53 \mu \mathrm{V})$, and $\mathrm{CzCc}($ mean $0.53 \mu \mathrm{V})$.

\section{Direct Peroneal Nerve SEP Montages Comparison}

Direct comparison of the montages showed similar results for intraoperative peroneal nerve SEPs. CiCc and $\mathrm{CzCc}$ showed clear advantage over $\mathrm{CzFz}$ in all five categories: $\mathrm{CiCc}$ presented with better traces than $\mathrm{CzFz}$ in $16.7 \%$ more of the IOMs for clarity, was $10.2 \%$ more repeatable, showed larger traces in $7.1 \%$ more of the IOMs, was more consistent in $14.7 \%$ more of the IOMs and presented with less noise in even $42.9 \%$ more of the IOMs, as shown in the lower part of Table 3.

$\mathrm{CzCc}$ recorded better traces than $\mathrm{CzFz}$ in even $34 \%$ more considering clarity, was more repeatable $28.2 \%$, showed larger amplitudes $21.2 \%$ more often, stayed more consistent throughout the operation in $27.5 \%$ more of the IOMs and presented with less noise $35.3 \%$ more often.

When compared with the tibial nerve recordings where differences between $\mathrm{CiCc}$ and $\mathrm{CzCc}$ were relatively minor, peroneal nerve SEP montage $\mathrm{CzC}$ had a very clear advantage over the $\mathrm{CiCc}$ montage. $\mathrm{CzCc}$ traces showed better clarity, larger amplitude, better repeatability, and consistency, 23.7, 14.1, 24.3, and 26.3\%, respectively, more frequent than $\mathrm{CiCc}$ recordings. However, only the noise level was significantly higher in $\mathrm{CzCc}$ traces and $\mathrm{CiCc}$ picked up less noise in $9.6 \%$ more of the IOMs. All differences (with the exception of size difference) between $\mathrm{CzFz}$ and $\mathrm{CiCc}$ were significant. 
TABLE 3 | Direct comparison of the montages $\mathrm{CzFz}, \mathrm{CiCc}$, and $\mathrm{CzCc}$ in five categories.

\begin{tabular}{|c|c|c|c|c|}
\hline \multirow{18}{*}{$\begin{array}{l}\text { Tibial } \\
(n=300)\end{array}$} & & CzFz (\%) & CiCc (\%) & Both equal (\%) \\
\hline & Clarity & $27.7 \pm 0.2$ & $32.6 \pm 0.1$ & 39.7 \\
\hline & Repeatability & $17.3 \pm 0.2$ & $37.0 \pm 0.1$ & 45.7 \\
\hline & Size & $18.3 \pm 0.2$ & $44.3 \pm 0.2$ & 37.3 \\
\hline & Consistency & $14.7 \pm 0.2$ & $41.3 \pm 0.2$ & 44.0 \\
\hline & Noise & $3.7 \pm 0.2$ & $62.3 \pm 0.1$ & 34.0 \\
\hline & & CzFz (\%) & CzCc (\%) & Both equal (\%) \\
\hline & Clarity & $13.7 \pm 0.2$ & $51.3 \pm 0.1$ & 35.0 \\
\hline & Repeatability & $9.7 \pm 0.2$ & $38.7 \pm 0.1$ & 51.7 \\
\hline & Size & $26.3 \pm 0.2$ & $43 \pm 0.2$ & 30.7 \\
\hline & Consistency & $11.3 \pm 0.2$ & $34.7 \pm 0.2$ & 54.0 \\
\hline & Noise & $4.0 \pm 0.2$ & $53.0 \pm 0.1$ & 43.0 \\
\hline & & $\mathrm{CiCc}(\%)$ & CzCc (\%) & Both equal (\%) \\
\hline & Clarity & $11.0 \pm 0.2$ & $24.0 \pm 0.1$ & 65.0 \\
\hline & Repeatability & $11.3 \pm 0.2$ & $17.7 \pm 0.1$ & 71.0 \\
\hline & Size & $25.0 \pm 0.2$ & $30.0 \pm 0.2$ & 45.0 \\
\hline & Consistency & $18.0 \pm 0.2$ & $13.7 \pm 0.2$ & 68.3 \\
\hline & Noise & $12.0 \pm 0.1$ & $7.0 \pm 0.1$ & 81.0 \\
\hline \multirow{18}{*}{$\begin{array}{l}\text { Peroneal } \\
(n=156)\end{array}$} & & $\mathrm{CzFz}(\%)$ & CiCc (\%) & Both equal (\%) \\
\hline & Clarity & $25.0 \pm 0.3$ & $41.7 \pm 0.3$ & 33.3 \\
\hline & Repeatability & $18.6 \pm 0.3$ & $28.8 \pm 0.3$ & 52.6 \\
\hline & Size & $28.8 \pm 0.3$ & $35.9 \pm 0.3$ & 35.3 \\
\hline & Consistency & $16.7 \pm 0.3$ & $31.4 \pm 0.3$ & 51.9 \\
\hline & Noise & $1.9 \pm 0.3$ & $44.8 \pm 0.2$ & 53.2 \\
\hline & & CzFz (\%) & $\mathrm{CzCc}$ & Both equal \\
\hline & Clarity & $14.1 \pm 0.3$ & $48.1 \pm 0.3$ & 37.8 \\
\hline & Repeatability & $11.5 \pm 0.3$ & $39.7 \pm 0.3$ & 48.7 \\
\hline & Size & $17.9 \pm 0.3$ & $39.1 \pm 0.3$ & 42.9 \\
\hline & Consistency & $9.6 \pm 0.3$ & $37.1 \pm 0.3$ & 53.2 \\
\hline & Noise & $3.8 \pm 0.3$ & $39.1 \pm 0.3$ & 57.1 \\
\hline & & $\mathrm{CiCc}(\%)$ & CzCc (\%) & Both equal (\%) \\
\hline & Clarity & $10.3 \pm 0.3$ & $34.0 \pm 0.3$ & 55.8 \\
\hline & Repeatability & $5.8 \pm 0.3$ & $30.1 \pm 0.3$ & 64.1 \\
\hline & Size & $16.0 \pm 0.3$ & $30.1 \pm 0.3$ & 53.8 \\
\hline & Consistency & $7.7 \pm 0.3$ & $33.3 \pm 0.3$ & 58.9 \\
\hline & Noise & $12.2 \pm 0.2$ & $2.6 \pm 0.3$ & 85.3 \\
\hline
\end{tabular}

Percentages represent which montage was better, unless they are equal. In bold are all statistically significant comparisons.

\section{Direct Comparison of Baseline Recordings and Their Development during Monitoring (Quick Approach)}

After this detailed analysis of the first 150 patients, another 748 tibial SEP recordings and 390 peroneal SEPs recordings of the remaining 374 patients were assessed and their electrode montages $\mathrm{CzFz}, \mathrm{CiCc}$, and $\mathrm{CzCc}$ were ranked at baseline as well as according to their development throughout the surgery. Same approach was used on the first 150 patients and for general comparison of the usability and reliability of these three montages for IOM, all 524 patients were evaluated.

\section{Tibial Nerve SEP Montages Comparison}

This direct comparison showed for the 1048 tibial nerve SEPs recordings that $\mathrm{CiCc}$ presented with better traces twice as often as $\mathrm{CzF}$. $\mathrm{CzCc}$ recorded better traces 4 times as often as $\mathrm{CzFz}$. Comparing the $\mathrm{CiCc}$ and $\mathrm{CzCc}$ montages, $\mathrm{CzCc}$ showed better recordings in $11.1 \%$ more of the IOMs. All of these results: $\mathrm{CzFz}$ vs. $\mathrm{CiCc}, \mathrm{CzFz}$ vs. $\mathrm{CzCc}$, and $\mathrm{CiCc}$ vs. $\mathrm{CzCc}$ were significant, as shown in Table 4.

$\mathrm{CzFz}$ was not monitorable in $142(13.5 \%)$ of all tibial SEP IOMs, while CiCc was non-monitorable in only $82 \mathrm{IOMs}(7.8 \%)$ and $\mathrm{CzCc}$ could not be recorded in $80 \mathrm{IOMs}$ (7.6\%). Thus, almost half of the cases with non-recordable $\mathrm{CzFz}$ traces became monitorable by using $\mathrm{CiCc}$ and $\mathrm{CzCc}$ montages. Out of the $142 \mathrm{IOMs}$ where no trace could be recorded over $\mathrm{CzFz}, 79$ traces (55.6\%) turned out to be monitorable by using $\mathrm{CiCc}$ montage and 75 traces $(52.8 \%)$ by using $\mathrm{CzCc}$ montage. There also existed some cases in which $\mathrm{CzFz}$ was monitorable but $\mathrm{CiCc}$ (32 traces, 3.1\% of all IOMs) or $\mathrm{CzCc}$ (29 traces, $2.8 \%$ of all IOMs) could not be followed. In $0.9 \%$ of the tibial nerve recordings, as an extreme, $\mathrm{CzFz}$ showed a good quality trace at baseline (score 4 or 5), but turned non-monitorable (score 1) during the procedure, while $\mathrm{CiCc}$ and/or $\mathrm{CzCc}$ stayed monitorable and could be followed. So, overall, combining all three montages, $94.3 \%$ of all IOMs were monitorable.

\section{Peroneal Nerve SEP Montages Comparison}

Results for the 546 peroneal nerves showed the same trend: $\mathrm{CiCc}$ montage recorded almost double as often better traces than $\mathrm{CzFz}$ montage, $\mathrm{CzCc}$ was in over 30\% more of the IOMs better than $\mathrm{CzFz}$. Comparing $\mathrm{CiCc}$ and $\mathrm{CzCc}$ montages, $\mathrm{CzCc}$ presented with the better trace in $26.2 \%$ more IOMs than CiCc.

Peroneal SEPs were not monitorable in a much higher number of IOMs than tibial nerve recordings in every of the three montages. In over a third of all IOMs (37\%), CzFz was not monitorable. CiCc could not record traces in $26.2 \%$ and $\mathrm{CzCc}$ presented as non-recordable in $23.6 \%$ of the IOMs. This shows a number of IOMs in which CzFz montage was non-monitorable, the peroneal nerve recording continued to be monitorable by adding $\mathrm{CiCc}$ and $\mathrm{CzCc}$ montages. $\mathrm{CiCc}$ was able to provide monitoring for additional $83(40.8 \%)$ and $\mathrm{CzCc}$ for additional 78 (38.4\%) out of the 203 IOMs where no trace was recordable with CzFz. However, in some cases $\mathrm{CiCc}$ (42 traces) and $\mathrm{CzCc}$ (34 traces) were non-monitorable although $\mathrm{CzFz}$ could be used for IOM. As an extreme, in 2.6\% of the peroneal recordings, $\mathrm{CzFz}$ recorded a good quality trace at baseline (score over 3) but then deteriorated to a point where it was no longer monitorable while at least one of the other montages stayed monitorable.

\section{Correlation of Baseline Recordings and Development Throughout the Monitoring}

Our study also looked at predicting whether an IOM recording would have well-formed SEPs throughout the monitoring, based on the quality of the baseline recordings. When the ranking of the traces at baseline was compared with the development throughout the cases, we found only moderate correlation according to Pearson's correlation coefficient for tibial $(\mathrm{rCzFz}=0.59, \mathrm{rCiCc}=0.62, \mathrm{rCzCc}=0.60)$ and peroneal $(\mathrm{rCzFz}=0.58, \mathrm{rCiCc}=0.49, \mathrm{rCzCc}=0.46)$ nerve SEPs. This means that it cannot be predicted at baseline which montage will be best monitorable throughout the surgery, supported with an example in Figure 5. 


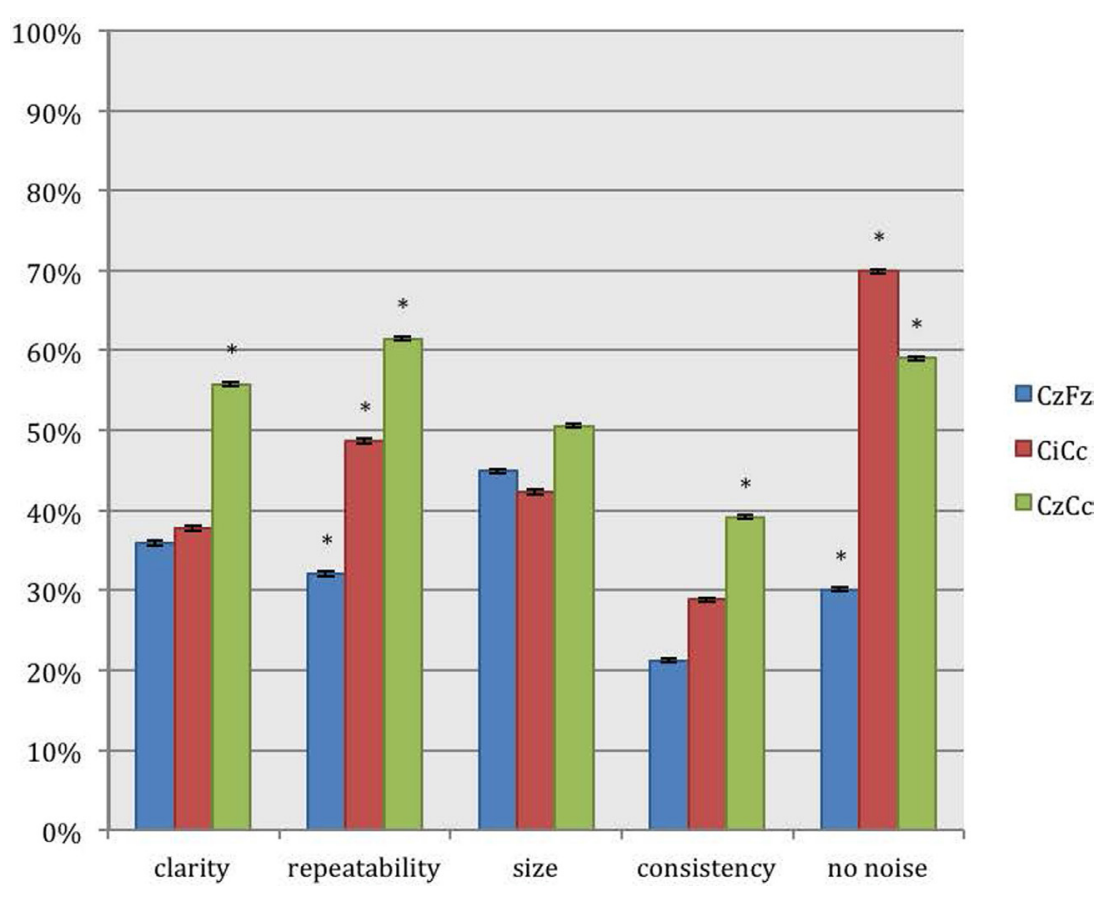

FIGURE 4 | Direct comparison of the montages $\mathrm{CzFz}, \mathrm{CiCc}$, and $\mathrm{CzCc}$ for peroneal nerve SEPs according to their score in the five categories: clarity, repeatability, size, consistency, and absence of noise. Asterisks $\left({ }^{*}\right)$ mark montages statistically significantly different from the other montages. All significance levels (Chi-square tests) $p<0.001$ with two exceptions: (a) consistency: CzCc vs. CzFz ( $p<0.001)$ and CzCc vs. CiCc ( $p=0.004)$; (b) absence of noise: CzFz vs. $\mathrm{CiCc}$ and $\mathrm{CzFz}$ vs. CzCc $(p<0.001)$; CiCc vs. CzCc $(p=0.003)$.

TABLE 4 | Overall pair wise comparison of three montages CzFz, CiCc and CzCc in all 524 patients.

\begin{tabular}{|c|c|c|c|c|c|c|c|c|c|c|}
\hline & & \multicolumn{3}{|c|}{ CzFz vs. CiCc } & \multicolumn{3}{|c|}{ CzFz vs. CzCc } & \multicolumn{3}{|c|}{ CiCc vs. CzCc } \\
\hline & & $\mathrm{CzFz}$ & $\mathrm{CiCc}$ & Equal & $\mathrm{CzFz}$ & $\mathrm{CzCc}$ & Equal & $\mathrm{CiCc}$ & $\mathrm{CzCc}$ & Equal \\
\hline \multirow[t]{2}{*}{ Tibial $(n=1048)$} & Better trace (\%) & 26.7 & 56.3 & 17.0 & 15.8 & 63.1 & 21.1 & 26.4 & 37.5 & 36.1 \\
\hline & Chi-square test & \multicolumn{3}{|c|}{$p<0.001^{*}$} & \multicolumn{3}{|c|}{$p<0.001^{*}$} & \multicolumn{3}{|c|}{$p<0.001^{*}$} \\
\hline \multirow[t]{2}{*}{ Peroneal $(n=546)$} & Better trace (\%) & 27.3 & 48.0 & 24.7 & 20.7 & 51.5 & 27.8 & 19.4 & 45.6 & 35.0 \\
\hline & Chi-square test & \multicolumn{3}{|c|}{$p<0.001^{*}$} & \multicolumn{3}{|c|}{$p<0.001^{*}$} & \multicolumn{3}{|c|}{$p<0.001^{*}$} \\
\hline
\end{tabular}

\section{DISCUSSION}

Despite the addition of other monitoring modalities, SEPs continue to be the standard modality used in IOM for various reasons, most importantly that they can be run frequently, and are not susceptible to overstimulation and neuromuscular blockade use. An improvement in their reliability and/or increase of the monitorable cases could be of great benefit for IOM.

The nerve most frequently recorded for SEP IOM is the tibial nerve, as it is the largest sensory nerve of the leg with the greatest area of representation in the somatosensory cortex. Therefore, it is generally used to assess the post central gyrus for brain surgery, the posterior column of the spinal cord in operations where the spinal cord is involved, and to assess the nerve roots in lumbar cases. This is generally achieved by monitoring SEPs using the $\mathrm{CzFz}$ electrode montage. Due to the paradoxical distribution of leg SEPs, it is recommended in recent guidelines to consider
$\mathrm{CiCc}$ and $\mathrm{CzCc}$ as additional electrode montages for intraoperative monitoring. According to our data, all montages are equally acceptable in around $17-36 \%$ of the IOMs. However, in the remainder of the IOMs where the patient had poor recordings with small amplitudes and/or delayed latency, more reliable monitoring is needed in order to detect true changes. Our study shows that $\mathrm{CzCc}$ and $\mathrm{CiCc}$ montages seem to be more appropriate than CzFz, as shown in Tables $\mathbf{2}$ and $\mathbf{4}$.

Peroneal traces often have very small amplitudes so that the recordings become unstable during the time course of the operation. This is due to the peroneal nerve being a small nerve at the level of the ankle where we stimulate it. For lumbar spine surgery with instrumentation of the levels L5 and lower, we are especially concerned with foot drop and the peroneal nerve SEP should additionally be recorded. Monitoring peroneal nerves in addition to tibial nerves serves as redundancy check and provides higher reliability for IOM. If traces change simultaneously in both 


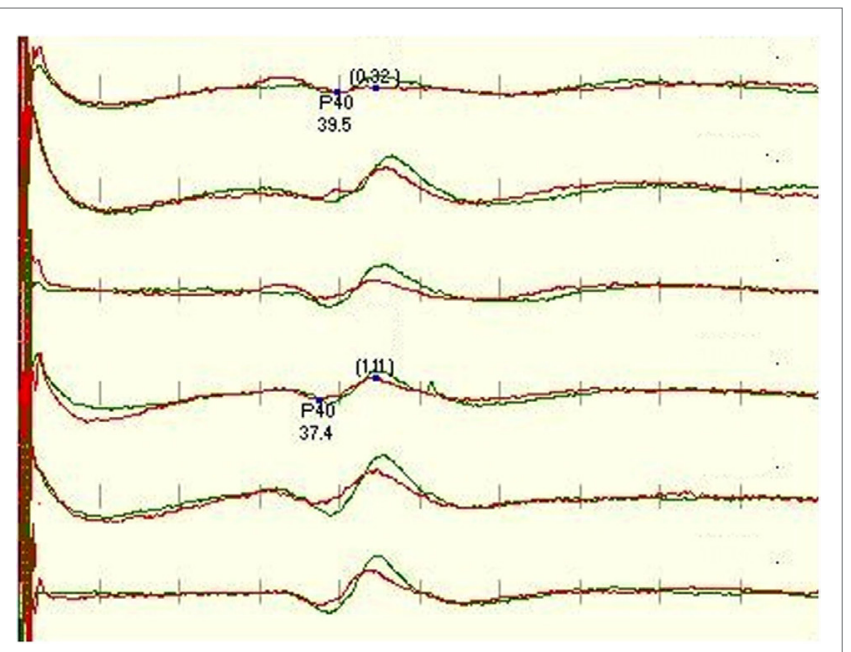

FIGURE 5 | Figure depicts a significant deterioration of the left leg-tibial nerve SEP trace recorded with $\mathrm{CzFz}$ montage (trace 1) with red being on-going recording compared with the green baseline recording. An acute reduction of $>50 \%$ in amplitude is a sign for possible neurological damage and typically needs to be reported to the surgeon. As the right tibial SEP CzFz (trace 4) recording stayed stable, this unilateral change implicates even more strongly that it is a true and reportable SEP change. However, this example shows that both transverse montages are minimally affected, especially $\mathrm{CiCc}$ (trace 2 ) which is rather stable. This stability in the other two montages ultimately prevents the neurophysiologist from interrupting the surgery and alarming the surgeon.

nerves, there is a very strong implication for a true positive event along the ascending pathway. Changes in only one nerve while the other one stays stable can be very specific, but makes the result more questionable. For the reasons named above, increasing the reliability of intraoperative peroneal SEP monitoring as well as reducing the number of non-monitorable cases would be of great value for IOM.

\section{$\mathrm{CiCc}$ and $\mathrm{CzCc}$ as Additional Derivations for IOM}

$\mathrm{CzFz}$ is historically used as the standard montage for intraoperative monitoring of leg SEPs. However, according to Table 2, which is an overall comparison of montages and their usability for reliable monitoring, this montage was actually found to provide quality recordings only in $58 \%$ of the tibial nerve SEPs and about $20 \%$ of the peroneal nerve SEPs. When the overall comparison of all montages against themselves was performed as depicted in Table 4, it corroborated our earlier findings that there are other montages more superior to $\mathrm{CzFz}$ for IOM. For tibial nerves, $\mathrm{CiCc}$ traces were equally as good as or better than $\mathrm{CzFz}$ traces in $73.3 \%$ of the IOMs, $\mathrm{CzCc}$ showed equally as good or better traces than $\mathrm{CzFz}$ in $84.1 \%$ of the IOMs. For peroneal $\mathrm{SEP}, \mathrm{CiCc}$ was equally as good as or better than $\mathrm{CzFz}$ in 72.7 , $\mathrm{CzCc}$ was better or equally as good as $\mathrm{CzFz}$ in $79.3 .1 \%$ of the IOMs. This shows that the monitorability of leg SEPs, particularly that of the peroneal nerve, can definitely be improved by additional montages, such as $\mathrm{CiCc}$, and especially by adding CzCc electrode montage.
This success rate for leg tibial SEP monitoring of $86.5 \%$ for $\mathrm{Cz}-\mathrm{Fz}$ montage is lower than the one described earlier by our group (20) where $93.4 \%$ of the leg SEPs recorded with $\mathrm{CzFz}$ montage showed a positive result. The population of patients evaluated in that study was predominantly brain surgery patients with no or little preoperative deficit. The patient population in this study consisted mainly of patients suffering from lumbar stenosis, cervical stenosis, radiculopathies, and scoliosis. All of the abovementioned conditions, if severe enough, can easily render SEPs non-recordable. Additionally, there is a possibility of polyneuropathy and foot swelling, which further complicates the monitoring, and can ultimately add to the non-monitorability of cases. Many patients had preoperative deficits and poorly formed traces to start off with at baseline. These cases are prone to show intraoperative fluctuations and it is crucial to be able to distinguish real changes from false positives. Improvement of the SEP recordings with additional electrode derivations is, therefore, of great benefit for IOM and ultimately of benefit for the patient. With addition of the two transverse montages ( $\mathrm{CiCc}$ and $\mathrm{CzCc}$ ), the success rate of tibial nerve SEP monitoring increased to $94.3 \%$, which is even slightly better than that in our previous study.

\section{Amplitude, Noise, and Consistency of SEP Monitoring}

We were not able to find a statistical difference between the sizes of amplitudes when comparing the three montages. These results somewhat vary from those of MacDonald et al. (21) who found their optimal derivation to be significantly larger than $\mathrm{CzFz}$ and, therefore, improve the signal-to-noise ratio (22). This may be due to the different patient population, as well as that we used standard electrode placements instead of lengthier search for maximum amplitudes. We evaluated many patients with preoperative neurologic deficit who presented with poor SEP traces. However, size evaluation alone is not enough to determine the optimal derivation. As long as the amplitudes are greater $(0.4 \mu \mathrm{V})$, clarity of waveform, noise, and especially consistency throughout the monitoring are even more important for IOM.

In the environment of the operating room, different sources of interference can affect the monitoring traces and make them unreliable or non-monitorable. Noise can occur at any point in time during the surgery, affecting different channels or montages differently, not only at the baseline. Usually, there is at least one montage that may pick up less noise than others. In our study, $\mathrm{CzFz}$ traces seem to be most prone to pick up these interferences during the surgery. Although $\mathrm{CiCc}$ and $\mathrm{CzCc}$ both showed less noise interference than $\mathrm{CzFz}$ in around $50-60 \%$ (tibial nerve) and $40-45 \%$ (peroneal nerve) of the cases, their traces can be affected as well. In our study, $\mathrm{CzCc}$ montage picked up noise only $5 \%$ more often than $\mathrm{CiCc}$ traces. Generally, SEP recordings are more likely to be affected by noise and interferences with larger distance between their recording and reference electrodes. This is a possible explanation for why $\mathrm{CzFz}$ and even more so when more frontal references such as $\mathrm{PFz}$ are used, tibial and peroneal nerve recordings appear noisier than $\mathrm{CiCc}$ and $\mathrm{CzCc}$ in so many cases. With $\mathrm{Cz}$ and $\mathrm{Cc}$ being slightly less apart than $\mathrm{Ci}$ and $\mathrm{Cc}$ electrodes, it remains unexplained why $\mathrm{CiCc}$ is the montage with 
the slightly better results according to noise level. It may, however, have to do with the generator orientation between recording and reference electrodes on one side and localization of the sensory leg area on the cortex in depth of the interhemispheric fissure. Changes in waveform, amplitude, and latency may also occur during the surgery. This is often due to fluctuations in anesthesia, temperature, or blood pressure. These inconsistencies surprisingly do not always occur over all montages. Therefore, one or more traces might decrease in amplitude or show delays in latency. It is difficult to predict, which montage will be the one that will change according to the baseline amplitude. Therefore, the largest amplitude montage does not necessarily predict the best consistency during the entire surgical procedure. As long as at least one other trace is not affected, changes in one montage do not need to be reported to the surgeon. It appears that $\mathrm{CzCc}$ is the least affected by all these changes. There is no good explanation for such often temporarily occurring changes. Possible causes include interference with the OR surrounding and anesthetic effects that lead to "wandering topography" of the optimal recording site. MacDonald suggested this to be caused by changes in the scalp topography due to intraoperative brain shifts of the cerebral volume and location (23). This suggestion does not explain the changes seen in our population, as they did not contain any craniotomies.

\section{The Importance of Multiple Montages, Especially $\mathrm{CzCc}$ for SEP Recordings in IOM}

Adding other montages for monitoring can decrease the number of false positives. Tibial CiCc and CzCc were around 15-20\% more reliable than $\mathrm{CzFz}$ recordings. For peroneal SEPs, CiCc showed $7.6 \%$ more consistent traces than $\mathrm{CzFz}$ and $\mathrm{CzCc}$ showed even better consistency than $\mathrm{CiCc}$ in another $17.9 \%$ of cases. Furthermore, we were able to lower the number of nonmonitorable cases by $\sim 50 \%$ for tibial traces and $20 \%$ for peroneal traces. This was achieved by adding $\mathrm{CiCc}$ and $\mathrm{CzCc}$ montages to the standard $\mathrm{CzFz}$ montage. $\mathrm{CiCc}$ seems to pick up the least amount of noise, while $\mathrm{CzCc}$ is usually the sturdiest trace. Although we were able to show that $\mathrm{CiCc}$ and $\mathrm{CzCc}$ traces are superior to the ones recorded by $\mathrm{CzFz}$ in many cases, the best electrode montage for a particular surgery can vary individually. There is only moderately significant correlation between baseline and development throughout the IOM. This can sometimes make it very difficult to predict at baseline which of the traces will stay most consistent or pick up the least amount of noise. Therefore, it is important to have more electrode montages for cortical recording from baseline on, and follow them all. Adding more channels and montages can help improve monitoring by filling gaps of inconsistency in other traces.

It is very common at many centers to use only one montage of electrodes for intraoperative cortical SEP recording. Oftentimes, subcortical montages are added for higher intraoperative reliability since these traces are generally less affected by anesthetics, blood pressure, and changes in temperature. Subcortical montage can be used for monitoring of spinal surgeries such as we routinely record, while they are not useful for monitoring of cranial cases as they are below the level of surgery and could be used only for monitoring of proper stimulation input. There is no question that multiple channel recordings provide more information and, therefore, higher reliability for intraoperative SEP monitoring. Although cervical traces are usually well defined with reasonably large amplitudes for arm SEP recording, they are often not very clear for leg recordings. In many cases, this leaves the monitoring technician with one effective montage $\mathrm{CzFz}$ only, for IOM. Any change in this montage, therefore, results in having to alarm the surgeon. Adding more cortical montages gives more traces to be compared and more reliability throughout the case and can ultimately lead to calling less false-positive changes.

As we were able to show, adding transverse montages $(\mathrm{CiCc}$, $\mathrm{CzCc}$ ) improves IOM reliability, reduces the number of nonmonitorable cases, and therefore is expected to reduce calling for false positives. $\mathrm{CiCc}$ and $\mathrm{CzCc}$ proved to be more consistent throughout the procedures. This is especially important for monitoring patients who suffer from severe spinal stenosis and myelopathies. A spectrum of abnormalities during surgeries can be observed; from slight decrease to over more than $50 \%$ deterioration of $\mathrm{CzFz}$ montage traces and even to turning to non-recordable. As already discussed non-monitorable cases could be reduced by over $50 \%$ for tibial and around $20 \%$ for peroneal recording. Non-monitorable cases have a higher risk for complications than monitorable cases (24). Therefore, any and every increase in the number of monitorable cases is important. Our study showed how especially important this was for peroneal SEPs as its monitorability was improved from 53 to $74 \%$. Still there a small number of cases in which $\mathrm{CzFz}$ montage trace is recordable but $\mathrm{CiCc}$ and $\mathrm{CzCc}$ traces show poor or no response. These cases would be missed by choosing transverse derivations only for cortical SEP recording and omitting CzFz. Therefore, we suggest a combination of transverse and longitudinal montages for IOM, covering $\mathrm{CzFz}, \mathrm{CiCc}$, and $\mathrm{CzCc}$ if the number of channels available allows doing so.

If there is a restriction in channels, $\mathrm{CzCc}$ and $\mathrm{CzFz}$ montages should be recorded as $\mathrm{CzCc}$ proved to be the overall best trace of the three and $\mathrm{CzFz}$ as standard montage has proven its value for IOM over decades. Yet, if only one channel is available, $\mathrm{CzFz}$ should be replaced by $\mathrm{CzCc}$. CzCc is still better in most qualities than CiCc. A small body of evidence is starting to build naming $\mathrm{CzCc}$ a better single channel choice than $\mathrm{CzFz}$ (21-25). It might be sensible to omit the subcortical trace, when not satisfying or monitorable, and create space for an additional cortical montage recording. Ultimately, if more channels are available, multichannel recording of all three montages $\mathrm{CzFz}, \mathrm{CiCc}$, and $\mathrm{CzCc}$ with addition of cervical recording could and should be used throughout the case. More cases would become reliably monitorable and the number of non-monitorable cases would be reduced. This is of benefit for surgery and in patients' best interest.

As a result of the findings of the data analysis that has been presented in this report, our approach to performing intraoperative monitoring has changed. We now routinely follow $\mathrm{Cz}-\mathrm{Cc}$ as the primary montage throughout the surgery, and use the other two 
montages as backup. This is a departure from our prior practice of using $\mathrm{Cz}-\mathrm{Fz}$ as the primary montage.

\section{AUTHOR CONTRIBUTIONS}

$\mathrm{CH}, \mathrm{AL}$, and $\mathrm{AB}$ all made substantial contributions to the conception and design of this original research. $\mathrm{CH}$ performed the analysis of the data, whereas $\mathrm{CH}, \mathrm{AB}$, and $\mathrm{AL}$ performed the interpretation of the data. The work was drafted by $\mathrm{CH}$, and revised by $\mathrm{AB}, \mathrm{CH}$, and $\mathrm{AL}$ who are in agreement to be accountable with the final version to be published.

\section{REFERENCES}

1. Larson SJ, Sances S Jr. Evoked potentials in man. Neurosurgical applications. Am J Surg (1966) 111(6):857-61.

2. Nash CL, Brodkey JS, Croft TJ. A model for electronical monitoring of spinal cord function in scoliosis patients undergoing correction (abstract). J Bone Joint Surg Am (1972) 54:197-8.

3. Nash CL, Lorig RA, Schatzinger LA, Brown RH. Spinal cord monitoring during operative treatment of the spine. Clin Orthop Relat Res (1977) 12:100-5.

4. Macon JB, Polettu CE. Conducted somatosensory evoked potentials during spinal surgery. Part 1: control conduction velocity measurements. J Neurosurg (1982) 57(3):349-53. doi:10.3171/jns.1982.57.3.0354

5. Nuwer MR, Dawson E. Intraoperative evoked potential monitoring of the spinal cord: enhanced stability of cortical recordings. Electroencephalogr Clin Neurophysiol (1984) 59:318-24. doi:10.1016/0168-5597(84)90049-2

6. Cracco RQ. Spinal evoked response: peripheral nerve stimulation in man. Electroencephalogr Clin Neurophysiol (1973) 35(4):379-86. doi:10.1016/00134694(73)90195-8

7. Jones SJ, Small DG. Spinal and sub-cortical evoked potentials following stimulation of the posterior tibial nerve in man. Electroencephalogr Clin Neurophysiol (1978) 44(3):299-306. doi:10.1016/0013-4694(78)90305-X

8. Beric A, Prevec TS. The early negative potential evoked by stimulation of the tibial nerve in man. J Neurol Sci (1981) 50(2):299-306. doi:10.1016/0022510X(81)90175-1

9. Beric A, Prevec TS. Distribution of scalp somatosensory potentials evoked by stimulation of the tibial nerve in man. J Neurol Sci (1983) 59(2):205-14. doi:10.1016/0022-510X(83)90038-2

10. Rossini PM, Cracco RQ, Cracco JB, House WJ. Short latency somatosensory evoked potentials to peroneal nerve stimulation: scalp topography and the effect of different frequency filters. Electroencephalogr Clin Neurophysiol (1981) 52:540-52. doi:10.1016/0013-4694(81)91429-2

11. Vas GA, Cracco JB, Cracco RQ. Scalp-recorded short latency cortical and subcortical somatosensory evoked potentials to peroneal nerve stimulation. Electroencephalogr Clin Neurophysiol (1981) 52:1-8. doi:10.1016/0013-4694 (81)90182-6

12. Cruse R, Klem G, Lesser RP, Lueders H. Paradoxical lateralization of cortical potentials evoked by stimulation of the posterior tibial nerve. Arch Neurol (1982) 39:222-35. doi:10.1001/archneur.1982.00510160028005

13. Yamada T, Machida M, Kimura J. Farfield somatosensory evoked potentials after stimulation of the tibial nerve. Neurology (1982) 32:1151-8. doi:10.1212/ WNL.32.10.1151

14. Emerson RG, Pedley TA. Somatosensory evoked potentials. 2nd ed. In: Daly DD, Pedley TA, editors. Current Practice of Clinical Electroencephalography. New York: Raven Press (1990). p. 679-706.

15. Yamada T. Neuroanatomic substrates of lower extremity somatosensory evoked potentials. J Clin Neurophysiol (2000) 17(3):269-79. doi:10.1097/ 00004691-200005000-00005

\section{FUNDING}

There was no external funding required for this publication. This work was done in accordance with ethical standards and was performed at the NYU School of Medicine. There was no IRB approval needed for this study, as it involved reviewing the traces without any patient identifiers that were obtained during routine protocols performed for neurophysiologic intraoperative monitoring, for which there was routine consent given for the instrumented fusion performed with intraoperative neurophysiologic monitoring.

16. Terada K, Umeoka S, Baba K, Sakura Y, Usui N, Matasuda K, et al. Generators of tibial nerve somatosensory evoked potential: recorded from the mesial surface of the human brain using subdural electrodes. J Clin Neurophysiol (2009) 26(1):13-6. doi:10.1097/WNP.0b013e3181969043

17. Nuwer MR, Daube J, Fischer C, Schramm J, Yingling CD. Neuromonitoring during surgery. Report of an IFCN committee. Electroencephalogr Clin Neurophysiol (1993) 87(5):263-73. doi:10.1016/0013-4694(93)90179-Y

18. Toleikis JR; American Society of Neurophysiological Monitoring. Intraoperative monitoring using somatosensory evoked potentials. A position statement by the American Society of Neurophysiological Monitoring. J Clin Monit Comput (2005) 19(3):241-58. doi:10.1007/s10877-005-4397-0

19. AmericanClinicalNeurophysiologySociety.Guideline9A:guidelinesonevoked potentials. J Clin Neurophysiol (2006) 23(2):125-37. doi:10.1097/00004691200604000-00010

20. Chen X, Sterio D, Ming X, Para DD, Butusova M, Tong T, et al. Success rate of motor evoked potentials for intraoperative neurophysiological effects of age, lesion location, and preoperative neurologic deficits. J Clin Neurophysiol (2007) 24(3):281-5. doi:10.1097/WNP.0b013e31802ed2d4

21. MacDonald DB, Stigsby Z, Al Zayed Z. A comparison between derivation optimization and $\mathrm{Cz}-\mathrm{FPz}$ for posterior tibial P37 somatosensory evoked potential intraoperative monitoring. Clin Neurophys (2004) 115:1925-30. doi:10.1016/j.clinph.2004.03.008

22. MacDonald DB, Al Zayed Z, Stigsby B. Tibial somatosensory evoked potential intraoperative monitoring: recommendations based on signal to noise ratio analysis of popliteal fossa, optimized P37, standard P37, and P31 potentials. Clin Neurophys (2005) 116:1858-69. doi:10.1016/j.clinph.2005.04.018

23. MacDonald DB. Individually optimizing posterior tibial somatosensory evoked potential P37 scalp derivations for intraoperative monitoring. J Clin Neurophysiol (2001) 18(4):364-71. doi:10.1097/00004691-200107000-00008

24. Thuet, Padberg AM, Raynor BL, Bridwell KH, Riew KD, Taylor BA, et al. Increased risk of postoperative neurologic deficit for spinal surgery patients with unobtainable intraoperative evoked potential data. Spine (2005) 30(18):2094-103. doi:10.1097/01.brs.0000178845.61747.6a

25. Miura T, Sonoo M, Shimizu T. Establishment of standard values for the latency, interval and amplitude parameters of tibial nerve somatosensory evoked potentials (SEPs). Clin Neurophys (2003) 114:1367-78. doi:10.1016/ S1388-2457(03)00094-4

Conflict of Interest Statement: The authors declare that the research was conducted in the absence of any commercial or financial relationships that could be construed as a potential conflict of interest.

Copyright (C) 2016 Hanson, Lolis and Beric. This is an open-access article distributed under the terms of the Creative Commons Attribution License (CC BY). The use, distribution or reproduction in other forums is permitted, provided the original author(s) or licensor are credited and that the original publication in this journal is cited, in accordance with accepted academic practice. No use, distribution or reproduction is permitted which does not comply with these terms. 